\title{
Actin filaments participate in the relocalization of phosphatidylinositol 3-kinase to glucose transporter-containing compartments and in the stimulation of glucose uptake in 3T3-L1 adipocytes
}

\author{
Qinghua WANG*, Philip J. BILAN*, Theodoros TSAKIRIDIS*, Aleksander HINEK† and Amira KLIP*1 \\ *Programme in Cell Biology, The Hospital for Sick Children, 555 University Avenue, Toronto, Ontario M5G 1X8, Canada, and †Programme in Cardiovascular Research, \\ The Hospital for Sick Children, 555 University Avenue, Toronto, Ontario M5G 1X8, Canada
}

Insulin stimulates the rate of glucose uptake into muscle and adipose cells by translocation of glucose transporters from an intracellular storage pool to the plasma membrane. This event requires the prior activation of phosphatidylinositol 3-kinase (PI 3-kinase). Here we report that insulin causes an increase in wortmannin-sensitive PI 3-kinase activity and a gain in the enzyme's regulatory and catalytic subunits $\mathrm{p} 85 \alpha$ and $\mathrm{p} 110 \beta$ (but not $\mathrm{p} 110 \alpha$ ) in the intracellular compartments containing glucose transporters. The hormone also caused a marked reorganization of actin filaments, which was prevented by cytochalasin D. Cytochalasin D also decreased significantly the insulin-dependent association of PI 3-kinase activity and the levels of insulin receptor substrate (IRS)-1, p85 $\alpha$ and $\mathrm{p} 110 \beta$ with immunopurified GLUT4-containing compartments. In contrast, the drug did not alter the insulin-induced tyrosine phosphorylation of IRS-1, the association of PI 3-kinase with IRS-1, or the stimulation of PI 3kinase by insulin in anti-(IRS-1) or anti-p85 immunoprecipitates from whole cell lysates. Cytochalasin D, and the chemically unrelated latrunculin B, which also inhibits actin filament reassembly, prevented the insulin stimulation of glucose transport by approx. $50 \%$. Cytochalasin $\mathrm{D}$ decreased by about one-half the insulin-dependent translocation to the plasma membrane of the GLUT1 and GLUT4 glucose transporters. The results suggest that the existence of intact actin filament is correlated with the full recruitment of glucose transporters by insulin. The underlying function of the actin filaments might be to facilitate the insulinmediated association of the p85-p110 PI 3-kinase with glucosetransporter-containing compartments.

\section{INTRODUCTION}

Insulin binding activates the tyrosine kinase of its receptor, leading to the rapid phosphorylation of a growing number of proteins characterized by the insulin receptor substrate (IRS) family and Shc. This triggers the activation of several signalling pathways, on the one hand the Ras/mitogen-activated protein kinase cascade of serine/threonine protein kinases, and on the other hand a phosphatidylinositol 3-kinase (PI 3-kinase) that phosphorylates phosphoinositides in the D-3 position. This insulin-regulated PI 3-kinase consists of the regulatory p $85 \alpha$ subunit [1], which binds to IRS-1, and a catalytic p110 subunit. Several lines of evidence suggest that PI 3-kinase is required for the stimulation of glucose transport. Two different inhibitors of this enzyme block the insulin-mediated increase in glucose uptake in fat and muscle cells [2-5]. In addition, insulin cannot stimulate glucose uptake in cells microinjected or transiently transfected with a dominant negative mutant of the $\mathrm{p} 85$ subunit that fails to bind the p110 subunit [5,6]. Furthermore, transient overexpression or microinjection of a wild-type or a constitutively activated form of the p110 catalytic subunit of PI 3-kinase in 3T3-L1 cells [7,8] and rat adipocytes [9] was sufficient to stimulate the translocation of GLUT4 glucose transporters to the cell surface.

In spite of the accepted requirement for PI 3-kinase enzymic activity in the regulation of glucose uptake, stimulation of this enzyme might not be sufficient to increase glucose transport. Indeed, the exposure of fat and muscle cells to platelet-derived growth factor (PDGF) $[10,11]$ and the addition of interleukin 4 to muscle cells transfected with interleukin 4 receptors [10] leads to robust stimulation of PI 3-kinase activity with a failure to stimulate glucose transport through translocation of GLUT4 transporters [10,11]. Moreover, the introduction of short peptides mimicking regions of IRS-1 that bind to and activate PI 3-kinase does not mimic the action of insulin on glucose transport [12].

The above studies suggest that mechanisms additional to the activation of PI 3-kinase are necessary for the stimulation of glucose uptake by insulin. It was recently reported that the cellular localization of the PI 3-kinase catalytic activity of 3T3$\mathrm{L} 1$ adipocytes changes in response to insulin, decreasing in the cytosol and increasing in intracellular membranes $[11,13,14]$. More specifically, PI 3-kinase activity was shown to increase in intracellular compartments containing GLUT4 (also called GLUT4 vesicles) within a few minutes of exposing adipocytes to insulin [15].

Insulin induces the reorganization of actin filaments in a variety of cells. This is best exemplified by the insulin-induced formation of membrane ruffles in Swiss 3T3 fibroblasts [16] and L6 myotubes [17] and formation of actin-containing stress fibres in fibroblasts [18] and rat mesangial cells [19]. We have recently reported that an intact actin network is required for the stimulation of glucose transport and the translocation of transporters in muscle cells [17]. Here we hypothesize that actin filaments might facilitate the activation or translocation of PI 3-kinase to the GLUT4-containing vesicles. To test this hypothesis we examine whether insulin causes changes in actin organization in 3T3-L1 adipocytes and whether a decrease in actin filaments affects the stimulation of glucose uptake and transporter trans-

Abbreviations used: CD, cytochalasin D; IRS, insulin receptor substrate; LDM, low-density microsomes; LB, latrunculin B; PDGF, platelet-derived growth factor; PI, phosphatidylinositol; PM, plasma membranes.

To whom correspondence should be addressed (e-mail amira@sickkids.on.ca). 
location. We also analyse whether actin filament disassembly affects insulin signalling, specifically the phosphorylation of IRS-1, the binding of p85 to IRS-1 and the activation and localization of PI 3 kinase.

\section{MATERIALS AND METHODS}

\section{Materials}

Dulbecco's modified Eagle's medium, calf serum, fetal bovine serum and antibiotic/antimycotic solution were purchased from Gibco/BRL (Burlington, ON, Canada). Cytochalasin D (CD) and cytochalasin B, 2-deoxy-D-glucose, paraformaldehyde, polyacrylamide and anti-(mouse $\mathrm{IgG}$ ) were obtained from Sigma Chemical Co. (St. Louis, MO, U.S.A.). Latrunculin B (LB) was obtained from BioMol (Plymouth Meeting, PA, U.S.A.). 2-Deoxy-D- $\left[{ }^{3} \mathrm{H}\right]$ glucose was purchased from Dupont/NEN (Boston, MA, U.S.A.). Human insulin was obtained from Eli Lilly Co. (Scarborough, ON, Canada). L- $\alpha$-Phosphatidylinositol (PI) was from Avanti Polar Lipids (Atlanta, GA, U.S.A.). Polyclonal antibodies against GLUT1, GLUT4 and monoclonal antibody against GLUT4 (1F8) were purchased from East Acres (Southbridge, MA, U.S.A.). Monoclonal antibody against the $\alpha 1 \mathrm{Na}^{+} / \mathrm{K}^{+}$-ATPase subunit (hybridoma culture supernatant McK1) was a gift from Dr. K. J. Sweadner (Harvard University, Boston, MA, U.S.A.) [20]. Polyclonal antibodies against IRS-1 and against $\mathrm{p} 85$, and monoclonal anti-phosphotyrosine antibody conjugated to agarose beads were purchased from Upstate Biotechnology (Lake Placid, NY, U.S.A.). In some experiments an IRS-1 antibody raised against the C-terminus of rat IRS-1 was used that was kindly provided by Dr. C. R. Kahn (Joslin Diabetes Center, Boston, MA, U.S.A.). Polyclonal antibodies to p1 $10 \alpha$ and p $110 \beta$ were from Santa Cruz Biotechnologies (Santa Cruz, CA, U.S.A.). ${ }^{125}$ I-Protein A and ${ }^{125}$ I-sheep anti-(mouse $\mathrm{IgG}$ ) were obtained from ICN (St. Laurent, Quebec, Canada). Sepharose conjugated to Protein A and Protein G were purchased from Pharmacia (Uppsala, Sweden). Rhodamine-labelled phalloidin was purchased from Molecular Probes (Eugene, OR, U.S.A.).

\section{Cell culture and cytochalasin D (CD) or LB treatments}

3T3-L1 fibroblasts were differentiated into adipocytes, as reported [21]. Cells were grown in 12-well plates for glucose transport assays, in 6-well dishes with coverslips for fluorescence microscopy and in $10 \mathrm{~cm}$-diameter dishes for subcellular fractionation. Adipocytes were serum-deprived for $3 \mathrm{~h}$ before experimental manipulations and were routinely incubated without or with $2 \mu \mathrm{M}$ CD or $2 \mu \mathrm{M} \mathrm{LB}$ (where indicated) in serum-free Dulbecco's modified Eagle's medium for $3 \mathrm{~h}$ before analysis. For stimulation with insulin, cultures were incubated with $100 \mathrm{nM}$ insulin for the last $30 \mathrm{~min}$ of the serum depletion or CD (or the LB) incubation period unless otherwise indicated. The CD pretreatment time of $3 \mathrm{~h}$ was chosen because in previous studies with L6 muscle cells the intracellular actin filamentous network was disrupted by $\mathrm{CD}$ in a time-dependent manner, reaching a maximal effect after $2 \mathrm{~h}$ [17], and preliminary experiments with 3T3-L1 adipocytes indicated that $2 \mu \mathrm{M}$ CD had its maximal inhibitory effect on glucose transport by $2.5 \mathrm{~h}$.

\section{Visualization of actin in cultured cells}

Fully differentiated adipocytes were gently trypsin-treated for 30 seconds and replated at a low cell density onto glass coverslips and allowed to recover for $48 \mathrm{~h}$ before analysis by fluorescence microscopy (Leica fluorescence microscope). Serum-deprived cells were treated with $\mathrm{CD}$ and/or insulin for the last $5 \mathrm{~min}$ of the
CD incubation period, as described above, where indicated. Actin filaments and bundles were detected by fluorescence microscopy after fixed and permeabilized cells had been stained with rhodamine-phalloidin [17].

\section{Glucose transport}

Glucose transport in differentiated 3T3-L1 adipocytes (treated as described above) was performed as described previously [17,22], after rinsing the cells to remove preincubation components.

\section{Immunoprecipitations}

IRS-1 was immunoprecipitated essentially as described previously for muscle cells [17]. Briefly, adipocytes grown in $10 \mathrm{~cm}-$ diameter dishes treated with or without $\mathrm{CD}$ were incubated with or without $100 \mathrm{nM}$ insulin for $3.5 \mathrm{~min}$. The cells were rinsed rapidly with $\mathrm{PBS}$ and lysed with $1.5 \mathrm{ml}$ of lysis buffer $[1 \%$ (v/v) Triton X-100/100 mM NaF/10 mM Na $\mathrm{P}_{2} \mathrm{O}_{7} / 2.5 \mathrm{mM}$ EDTA/2 mM Na $\mathrm{VO}_{4} / 1 \mathrm{mM}$ PMSF/50 mM Hepes (pH 7.8)] and multiple passages through a size 27 syringe. The insoluble material was removed by centrifugation at $100000 \mathrm{~g}$ for $1 \mathrm{~h}$ at $4{ }^{\circ} \mathrm{C}$ and the supernatant was incubated with $4 \mu \mathrm{g}$ of anti-(IRS1) polyclonal antibody for $16 \mathrm{~h}$ at $4{ }^{\circ} \mathrm{C}$. Anti-p85 immunoprecipitations were performed essentially as described previously [4]. In brief, untreated, CD-pretreated insulin-stimulated adipocytes were washed once with PBS, twice with buffer A [137 mM $\mathrm{NaCl} / 1 \mathrm{mM} \quad \mathrm{MgCl}_{2} / 1 \mathrm{mM} \quad \mathrm{CaCl}_{2} / 100 \mu \mathrm{M} \quad \mathrm{Na}_{3} \mathrm{VO}_{4} / 20 \mathrm{mM}$ Hepes (pH 7.5)] and lysed in $1 \mathrm{ml}$ of buffer A containing $10 \%$ (v/v) glycerol, $1 \%$ (v/v) NP40 and 2 mM PMSF, by shaking on ice for $25 \mathrm{~min}$ at $4{ }^{\circ} \mathrm{C}$. The lysates were centrifuged for $10 \mathrm{~min}$ at $4{ }^{\circ} \mathrm{C}$ to remove DNA and other insoluble material, and the supernatants were incubated with $2 \mu$ l of the polyclonal anti-p85 for $16 \mathrm{~h}$ with rotation at $4{ }^{\circ} \mathrm{C}$. The immunocomplexes were bound to $10 \mathrm{mg}$ (in $100 \mu \mathrm{l}$ ) Protein A-Sepharose for $4 \mathrm{~h}$ by rotation at $4{ }^{\circ} \mathrm{C}$, pelleted by centrifugation, washed three times with PBS, boiled for 3 min in Laemmli sample buffer [23] and subjected to immunoblotting. Parallel immunoprecipitates of anti-p85 and anti-(IRS-1) were washed and subjected to PI 3kinase activity assay in vitro as described below.

\section{Immunoisolation of GLUT4 glucose-transporter-containing compartments (vesicles)}

Isolation of plasma membranes (PM) and low-density microsomes (LDM), and immunoblotting were performed as described previously [24]. GLUT4-containing vesicles were immunoisolated from the supernatant containing LDM and cytosol but depleted of PM and heavy microsomes as described previously [24,25], with the monoclonal anti-GLUT4 antibody (1F8) coupled to Protein G-Sepharose beads (Pharmacia). Non-immune mouse IgG coupled to Protein G-Sepharose was used as a control for the 1F8 immunoisolations. Any membrane that would bind non-specifically to the Sepharose matrix would be accounted for in this control. Vesicles bound to the beads were washed three times in PBS subjected to either SDS/PAGE followed by immunoblotting with the indicated antibodies or to PI 3-kinase activity assays.

\section{PI 3-kinase activity}

The PI 3-kinase activity assay was performed as described previously [4]. When the activity was measured directly on immunopurified GLUT4-containing vesicles, it is stated as PI kinase; when measured on anti-p85 immunoprecipitates or anti(IRS-1) immunoprecipitates from lysates, membranes or 

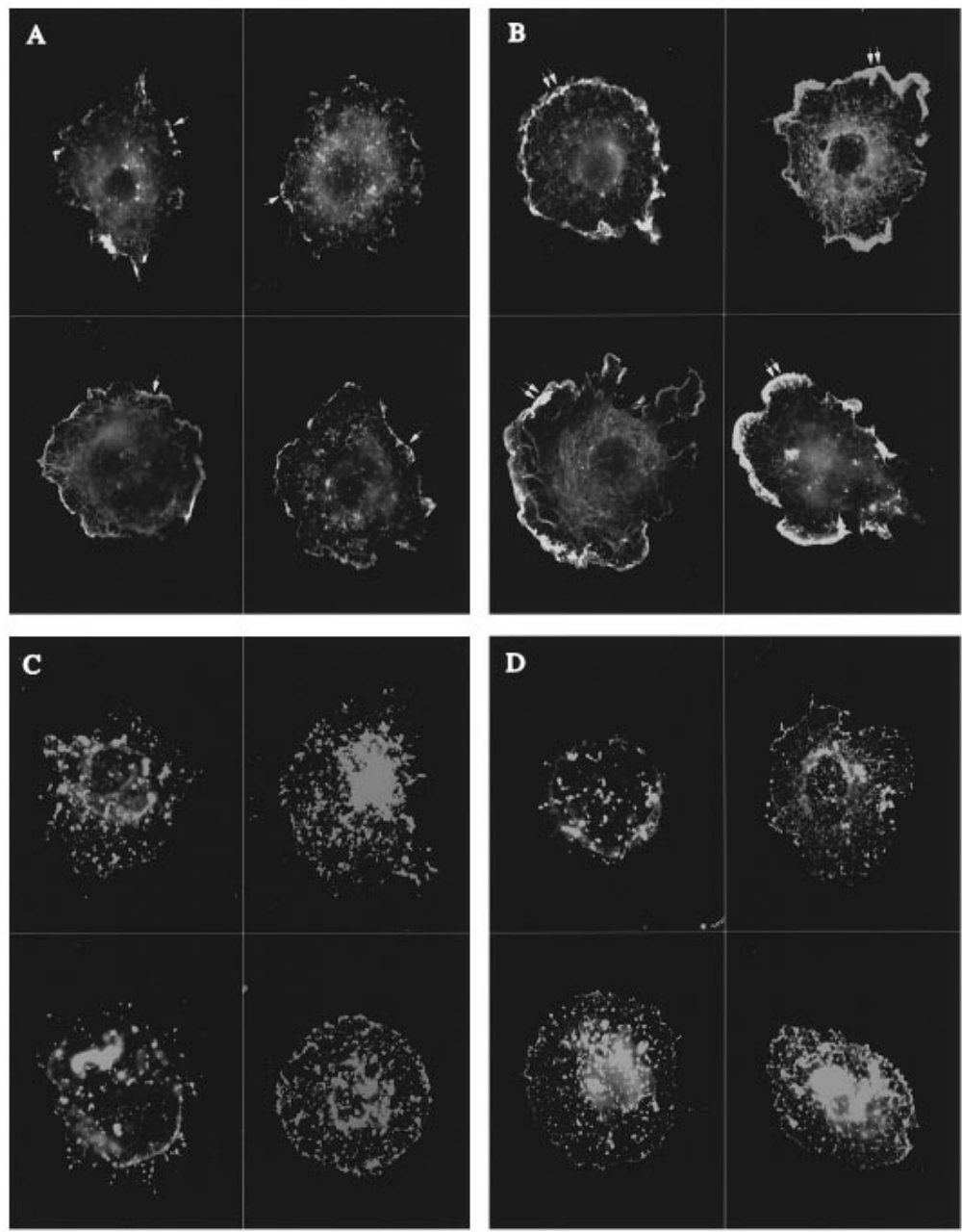

\section{Figure 1 Effects of insulin and CD on 3T3-L1 adipocyte actin filaments}

3T3-L1 adipocytes grown and differentiated on culture dishes were replated on glass coverslips where they were serum-depleted for $3 \mathrm{~h}$, pretreated with or without CD ( $2 \mu \mathrm{M})$ for $3 \mathrm{~h}$, then stimulated with or without $100 \mathrm{nM}$ insulin for the last 5 min of the incubation period. The samples were fixed and permeabilized as described in the Materials and methods section. Actin filaments were stained with rhodamine-labelled phalloidin. (A) Untreated adipocytes; (B) insulin-stimulated adipocytes; (C) CD-pretreated adipocytes; (D) CD-pretreated insulin-stimulated adipocytes. The single and double arrows point to membrane ruffles on untreated adipocytes and insulin-stimulated adipocytes respectively. These images of adipocytes are representative of those seen in three or four independent experiments; 20-30 single cells were evaluated in each experiment. Shown are for representative cells for each treatment.

GLUT4-containing vesicles, it is stated as PI 3-kinase. All samples were washed and incubated for 5 min with $20 \mu \mathrm{g}$ of PI. The reaction was started by the addition of $5 \mu 1$ of $1 \mathrm{mM}\left[\gamma^{-32} \mathrm{P}\right]$ ATP $(10 \mu \mathrm{Ci} / 5 \mu \mathrm{l})$ in $20 \mathrm{mM} \mathrm{MgCl}{ }_{2}$ and then stopped after $10 \mathrm{~min}$ by the addition of $20 \mu \mathrm{l}$ of $8 \mathrm{M} \mathrm{HCl}$. The lipids were extracted with $160 \mu 1$ of chloroform/methanol $(1: 1, \mathrm{v} / \mathrm{v})$. The lower phase $(50 \mu 1)$ was applied on an oxalate-pretreated Silica gel 60 plate, prebaked for $1 \mathrm{~h}$ at $110^{\circ} \mathrm{C}$, and was separated for $60 \mathrm{~min}$ by chromatography with chloroform/methanol/water/hydroxylamine (60:47:12:2, by vol.). The ${ }^{32} \mathrm{P}$-labelled phosphatidylinositol 3'-phosphate(s) signals on the silica plates were quantified by either using the Molecular Dynamics Phosphorimager system (Sunnyvale, CA, U.S.A.) or by scraping the radioactive spots off the silica followed by scintillation counting, as indicated.

\section{Statistics}

Where indicated, analysis of variance (ANOVA) or Student's $t$ test (paired) were applied. The paired $t$ test collects the differences between two conditions from separate experiments and analyses the mean of the differences to determine significance. Hence it was applied to experiments in which the controls were assigned a value of one. All the $P$ values from the statistical analyses are given in the Figure legends.

\section{RESULTS}

\section{Effects of insulin on the actin network of 3T3-L1 adipocytes}

Actin filaments undergo continuous assembly and disassembly in mammalian cells; the balance of these processes dictates the level of polymerized actin at any time. In unstimulated 3T3-L1 adipocytes, actin-containing structural elements were not apparent except for bundles of cortical actin filaments at the cell perimeter (Figure 1A, single arrow). Insulin (100 nM) elicited a rapid reorganization of cortical filaments, generating more pronounced membrane ruffles/lamellipodia within 5 min (Figure $1 \mathrm{~B}$, double arrow). $\mathrm{CD}$ is a fungal metabolite that caps the barbed (polymerizing) end of actin filaments. Thus exposure of 


\section{Table 1 Effect of CD and LB on insulin-stimulation of glucose transport}

Serum-deprived 3T3-L1 adipocytes were pretreated without or with $2 \mu \mathrm{M} \mathrm{CD}$ or $2 \mu \mathrm{M} \mathrm{LB}$ for $3 \mathrm{~h}$ with or without $100 \mathrm{nM}$ insulin during the last $30 \mathrm{~min}$ of the incubation period, as indicated. 2-Deoxy-D-glucose transport was subsequently determined for 5 min. Seven (CD) and three (LB) independent experiments were performed, each in triplicate (data given as means \pm S.E.M.). ${ }^{*} P<0.001$ (ANOVA) for insulin compared with CD plus insulin. $\uparrow P<0.003$ (ANOVA) for insulin compared with LB plus insulin.

\begin{tabular}{lll}
\hline & \multicolumn{2}{l}{$\begin{array}{l}\text { 2-Deoxy-D-glucose uptake } \\
\text { (pmol/mg per min) }\end{array}$} \\
\cline { 2 - 3 } Treatment & CD & \multicolumn{1}{c}{ LB } \\
\hline Untreated & $4.10 \pm 0.15$ & $2.99 \pm 0.18$ \\
Insulin & $29.9 \pm 1.96^{\star}$ & $17.7 \pm 0.51 \dagger$ \\
Drug & $3.76 \pm 0.26$ & $3.10 \pm 0.13$ \\
Drug + insulin & $18.4 \pm 1.17^{\star}$ & $9.17 \pm 0.34 \dagger$ \\
& & \\
\hline
\end{tabular}

cells to this drug for several hours is often required for a significant loss of actin filaments, by preventing filament reassembly in the course of their natural break down. Because actin depolymerization continues at an unaltered rate, actin filaments are dismantled within a few hours. In 3T3-L1 adipocytes, monitoring the effects of $\mathrm{CD}$ on the cytoskeleton was restricted mainly to its ability to disrupt the cortical actin bundles at the cell periphery. Actin filaments and cortical actin bundles were disrupted after a $3 \mathrm{~h}$ treatment with $2 \mu \mathrm{M}$ CD (Figure 1C). Cells pretreated with $\mathrm{CD}$ for $3 \mathrm{~h}$, subsequent to the exposure to insulin (Figure 1D), failed to produce actin reorganization (membrane ruffling) observed in the absence of CD. Overall, the results suggest that 3T3-L1 adipocytes contain actin filaments that are dynamically reorganized by insulin and that CD can substantially interfere with this reorganization.

\section{Effect of CD and LB on glucose transport}

The above results prompted us to examine whether polymerized actin is required for the stimulation of glucose uptake by insulin. Unlike cytochalasin B, a classical inhibitor of glucose transport, $\mathrm{CD}$ does not directly inhibit the glucose transporters at concentrations up to $2 \mu \mathrm{M}$ [17]. Control 3T3-L1 adipocytes or adipocytes preincubated with $\mathrm{CD}$, with or without subsequent

A
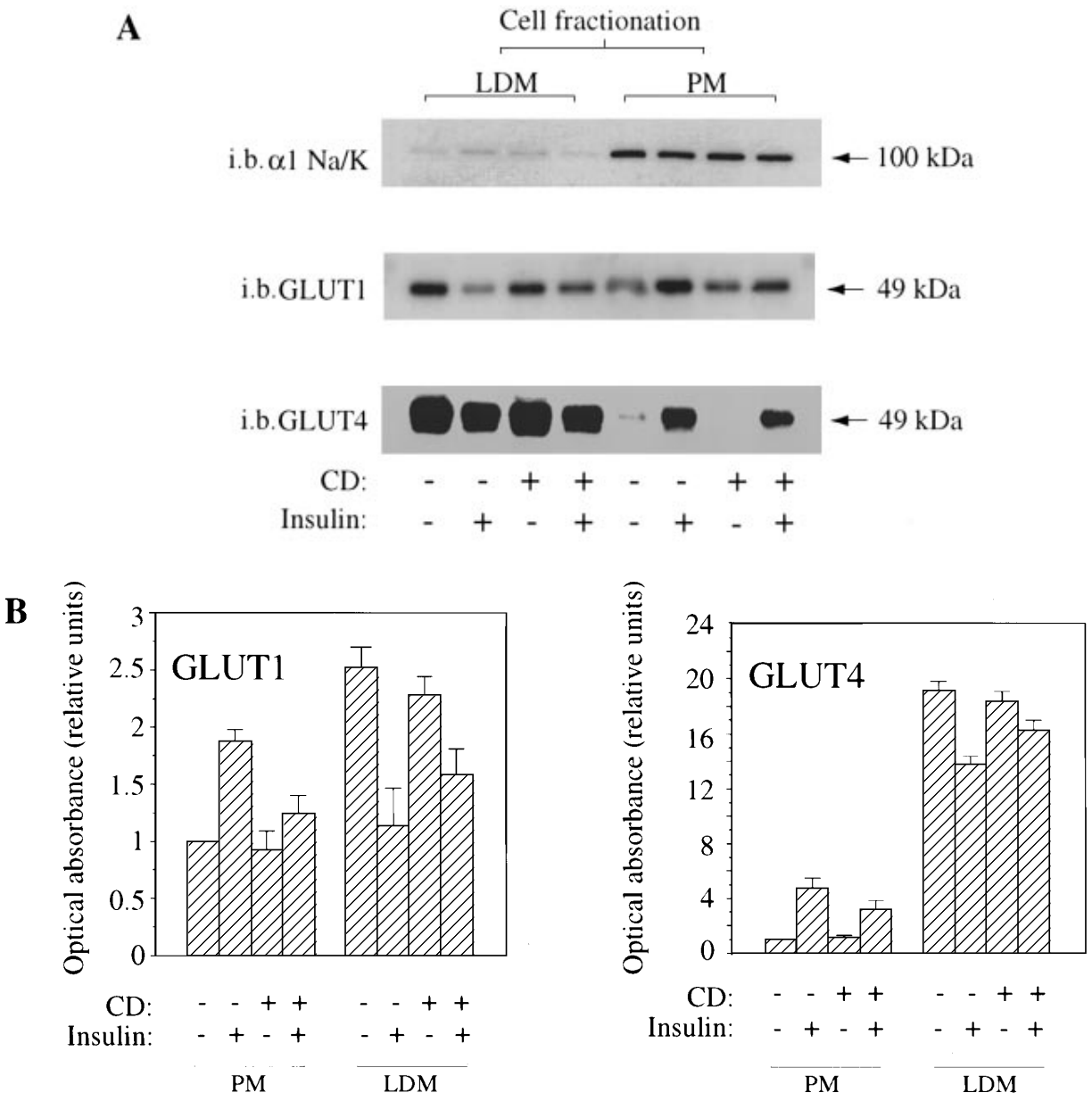

Figure 2 Effect of insulin and CD on intracellular distribution of glucose transporters

PM and LDM were isolated from untreated, insulin-stimulated, CD-pretreated or CD-pretreated insulin-stimulated cells. Membrane protein (20 $\mu \mathrm{g})$ of each fraction were resolved by SDS/PAGE and analysed by immunoblotting (i.b.). (A) Distribution of the PM marker $\alpha 1$ subunit of $\mathrm{Na}^{+} / \mathrm{K}^{+}$-ATPase, GLUT1 and GLUT4 glucose transporters (primary antibody dilutions were $1: 500,1: 1000$ and 1:1000 respectively). (B) Quantification of densitometrically scanned immunoblots of GLUT1 and GLUT4 transporters from six independent experiments. Values are means \pm S.E.M. expressed as the fold change relative to the PM fraction derived from untreated cells in the basal state. The insulin-dependent net gains ( $\Delta)$ in GLUT1 and GLUT4 in the PM of CD-pretreated cells were significantly different $(P<0.05$ and $P<0.02$ respectively) from those in untreated cells. The insulin-dependent net losses ( $\Delta)$ in GLUT1 and GLUT4 in the LDM of CD-pretreated cells were significantly different $(P<0.01$ and $P<0.05$ respectively) from those in untreated cells. 
insulin treatment, were assayed for 2-deoxy-D-glucose transport (Table 1). Insulin caused an 7.3-fold increase in glucose uptake in untreated 3T3-L1 adipocytes, which was decreased by $39 \%(P$ $<0.01)$ in CD-pretreated cells. This inhibition of insulinstimulated glucose transport was time dependent and peaked at $2.5 \mathrm{~h}$ of pretreatment with $2 \mu \mathrm{M} \mathrm{CD}$ (results not shown). Insulinstimulated glucose transport could not be inhibited further by using higher concentrations of $\mathrm{CD}$ because basal rates of glucose transport were also inhibited. Nevertheless the disassembly of actin filaments by CD coincided with a significant decrease in the stimulation of glucose transport by insulin.

LB is a marine macrolid that prevents actin polymerization by forming complexes with actin monomers and excluding them from assembly into existing actin filaments [26]. This drug is structurally different from CD and acts at a different point in the actin filament turnover but, like $\mathrm{CD}$, its action also results in actin disassembly. Table 1 indicates that maximally effective doses of LB $(2 \mu \mathrm{M})$ inhibited insulin-stimulated glucose transport by $48 \%(P<0.05)$, nearly the same magnitude as $\mathrm{CD}$, without affecting basal glucose transport.

\section{Effect of CD on glucose transporters}

The inhibition of glucose uptake could be due to a decrease in the translocation of glucose transporters to the cell surface, or to the inhibition of the intrinsic activity of the recruited transporters. Therefore we investigated the effect of actin filament disassembly on the distribution of the GLUT1 and GLUT4 glucose transporters that are expressed in these cells, both of which are mobilized to the cell surface in response to insulin [24,27]. By subcellular fractionation, PM and LDM were isolated from control and insulin-stimulated cells, each with or without CD pretreatment. Figure 2(A) shows the distribution of the glucose transporters in the isolated membrane fractions, in comparison with the distribution of a PM marker, the $\alpha 1$ subunit of $\mathrm{Na}^{+} / \mathrm{K}^{+}$ATPase. Whereas the distribution of this latter protein was not altered by any of the treatments, mobilization of the glucose transporters by insulin was markedly affected. The results of six independent experiments are summarized in Figure 2(B) and expressed as the changes in the levels of each glucose transporter relative to the basal level in the $\mathrm{PM}$, which for comparative purposes was set at 1.0. On its own, CD pretreatment did not alter the subcellular distribution of GLUT1 and GLUT4. Insulin caused the well-documented decrease in both GLUT1 and GLUT4 in the LDM, and concomitant increases in the amount of each transporter in the PM (GLUT1 and GLUT4 were elevated 1.9-fold and 4.8-fold by insulin in the PM respectively). Importantly, pretreatment with CD significantly decreased the subsequent insulin-induced translocation of both transporters from the LDM to the PM (GLUT1 and GLUT4 were elevated only 1.3 -fold and 3.3-fold in the PM by insulin after CD pretreatment respectively). The insulin effect was statistically different in CD-pretreated cells from that in control cells, for each transporter and for each membrane fraction (see legend to Figure 2). The magnitudes of the decreases in glucose transporter translocation were consistent with the decrease in glucose uptake caused by $\mathrm{CD}$. This suggests that cytoskeletal elements participated in the translocation of a significant portion of the internal pool of glucose transporters, by allowing insulin-dependent signals to arrive at the transporters and/or by allowing the primed vesicles to reach their target membrane.

\section{Effect of CD on early insulin signals}

The decrease in insulin-stimulated glucose uptake caused by CD suggested that actin filaments might participate in the trans-

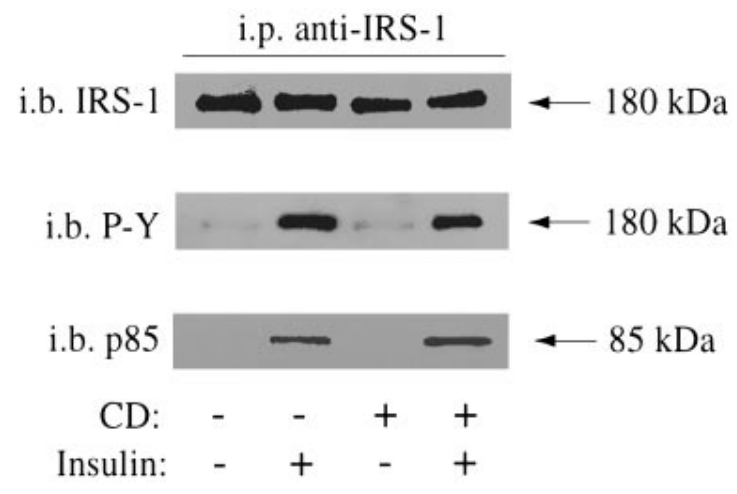

Figure 3 Effect of CD on insulin-stimulated tyrosine phosphorylation of IRS-1 and the association of IRS-1 with the p85 $\alpha$ subunit of PI 3-kinase

IRS-1 was immunoprecipitated (i.p.) from untreated, insulin-stimulated, CD-pretreated or CDpretreated insulin-stimulated adipocyte cell lysates. Anti-(IRS-1) immunoprecipitated protein complexes were resolved by SDS/PAGE and were immunoblotted (i.b.) with anti-(IRS-1), anti(P-Y), or anti-p85 $\alpha$ antibodies, and detected by enhanced chemiluminescence. One representative experiment of three is shown.

\section{Table 2 Effect of CD on the insulin stimulation of PI 3-kinase activity}

p85 $\alpha$ and IRS-1 proteins were immunoprecipitated from untreated, insulin-stimulated, CDpretreated (CD), or CD-pretreated insulin-stimulated adipocyte lysates. PI 3-kinase activity associated with anti-p85 $\alpha$ and anti-(IRS-1) immunoprecipitates was assayed as described in the Materials and methods section. Autoradiographs of TLC plates were scanned and quantified with a Molecular Dynamics Phosphorlmager system. The optical absorbance values of untreated cells were assigned a value of 1.0. The values obtained for all other conditions were expressed relative to untreated cells. The results are means \pm S.E.M. for four independent experiments. Insulin significantly elevated the enzymic activity of PI 3-kinase in the untreated or CD-pretreated cells respectively that co-purified with either p85 $\alpha$ immunoprecipitates $(P<0.02, P<0.01$, Student's paired $t$ test) or IRS-1 immunoprecipitates $(P<0.02, P<0.02$, Student's paired $t$ test). Significance levels: n.s., no significant difference for insulin compared with $C D$ plus insulin in the p85 $\alpha$ immunopurification (i.p.); * $P<0.05$ (Student's unpaired $t$ test) for insulin compared with CD plus insulin in the IRS-1 i.p.

\begin{tabular}{lll}
\hline & \multicolumn{2}{l}{ PI 3-kinase activity (relative to untreated) } \\
\cline { 2 - 3 } Treatment & p85 $\alpha$ i.p. & IRS-1 i.p. \\
\hline Untreated & 1.00 & 1.00 \\
Insulin & $1.66 \pm 0.12$ (n.s.) & $6.90 \pm 1.32^{\star}$ \\
CD & $1.22 \pm 0.28$ & $1.45 \pm 0.20$ \\
CD + insulin & $1.96 \pm 0.40$ (n.s.) & $13.2 \pm 3.77^{\star}$ \\
\hline
\end{tabular}

mission of signals from the insulin receptor to the glucose transporter-containing vesicles. We considered whether early signalling events in insulin action might be affected by $C D$, such as phosphorylation of IRS-1, the association of the $\mathrm{p} 85 \alpha$ subunit of PI 3-kinase with the phosphorylated IRS-1, and/or the activation of PI 3-kinase catalytic activity. Thus IRS-1 was immunoprecipitated from basal, insulin-stimulated, CD-pretreated and CD-pretreated insulin-stimulated adipocytes (Figure 3). Immunoprecipitates were resolved by SDS/PAGE and immunoblotted with anti-(IRS-1), anti-(P-Y) and anti-p85 $\alpha$ antibodies (Figure 3). Insulin caused the expected tyrosine phosphorylation of IRS- 1 and the association of $\mathrm{p} 85$ with IRS1. CD pretreatment did not alter the levels of IRS-I protein, its insulin-stimulated tyrosine phosphorylation or its association with the p85 $\alpha$ subunit of PI 3-kinase. Insulin significantly elevated 


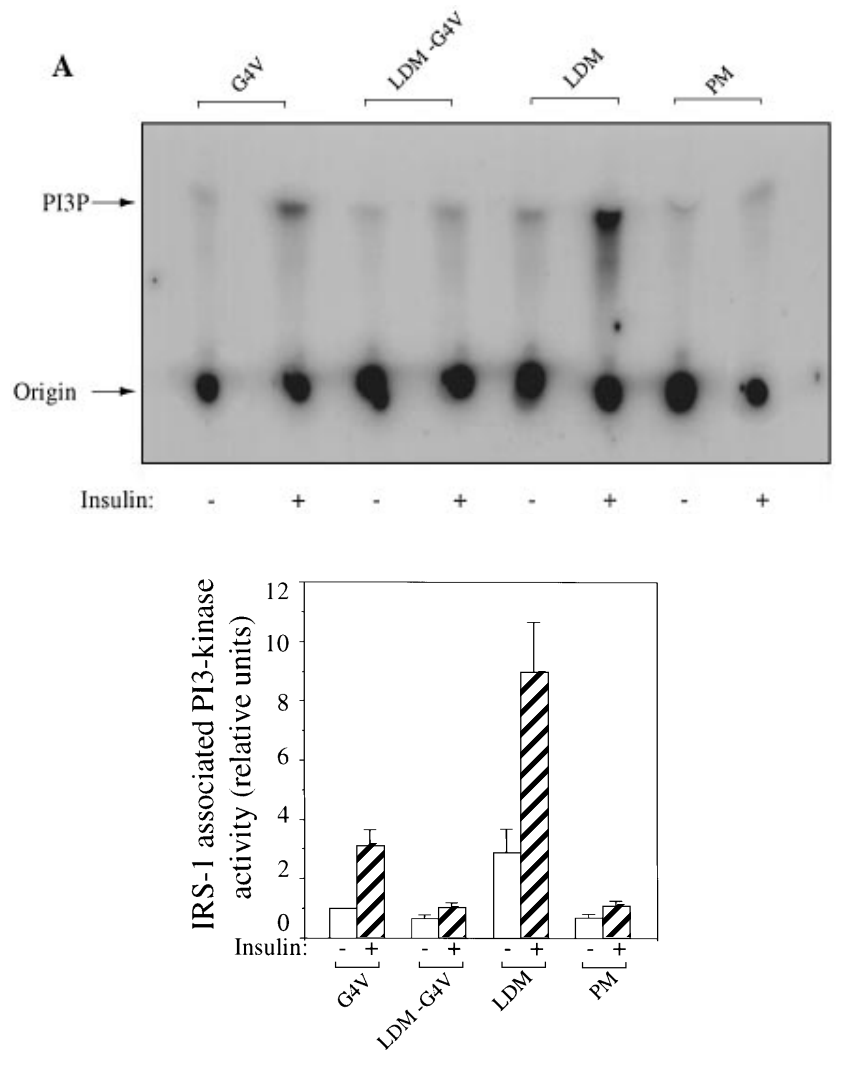

B
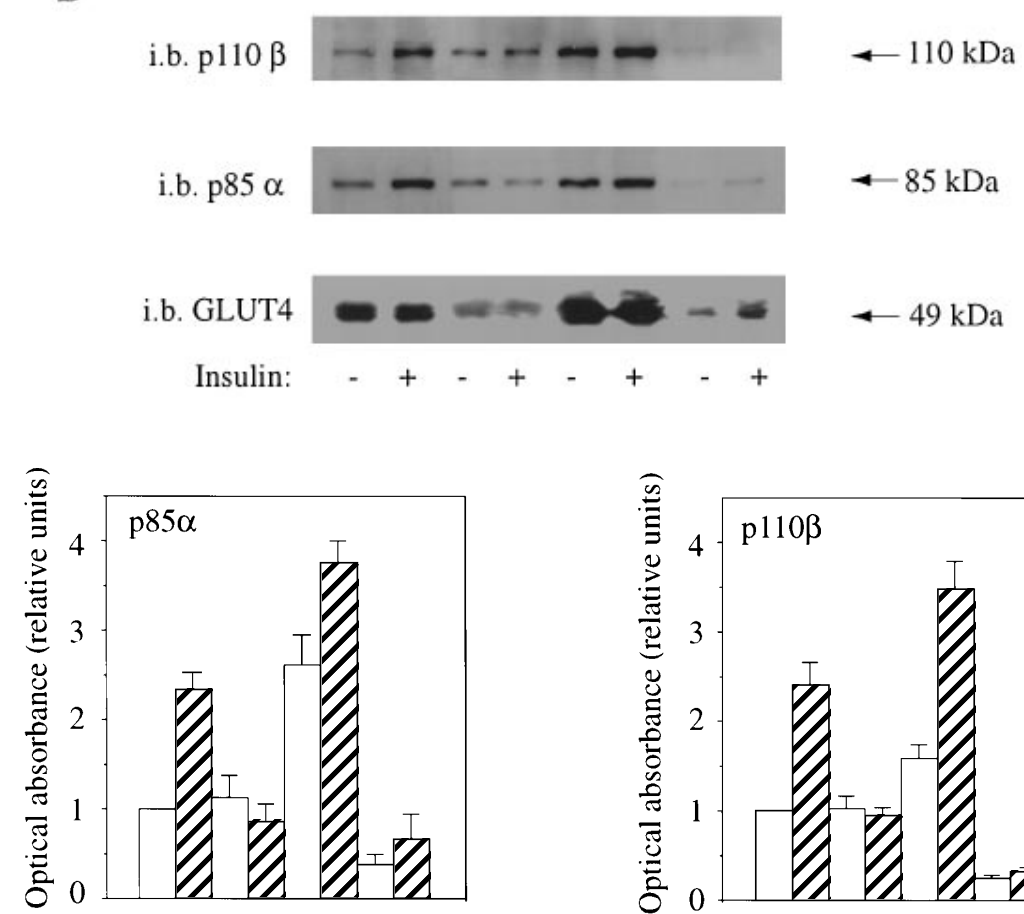

Insulin:
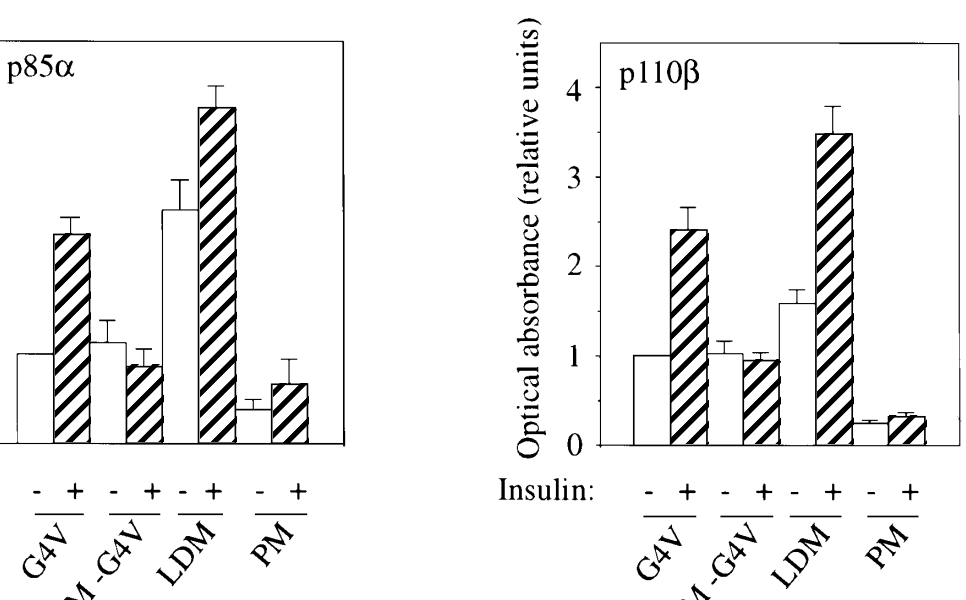

Figure 4 For legend see opposite page.

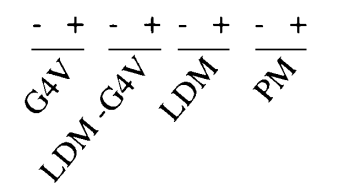


the enzymic activity of PI 3-kinase that co-purified with either p85 $\alpha$ immunoprecipitates (1.7-fold; $n=3, P<0.02$; Table 2$)$ or IRS-1 immunoprecipitates (6.9-fold; $n=4, P<0.02$; Table 2). In the presence of $\mathrm{CD}$, insulin still fully stimulated PI 3-kinase activity measured in p $85 \alpha$ immunoprecipitates (Table 2 ) or in IRS-1 immunoprecipitates (Table 2). In fact, insulin-stimulated PI 3-kinase activity associated with IRS-1 was higher in samples from CD-pretreated cells than from untreated cells.

\section{Effect of CD on PI 3-kinase activity, PI 3-kinase subunits and IRS-1 on GLUT4-containing vesicles}

The above results suggest that if there are defects in insulin signalling that arise from the disruption of the native cytoskeletal organization, they might lie downstream of the activation of PI 3-kinase. In 3T3-L1 adipocytes, the greatest portion of the insulin-stimulated PI 3-kinase activity associated with IRS-1 [13] or total phosphotyrosine proteins [14] is recovered in the LDM fraction. We surmised that the possible candidate step at which CD might act could be the activation or association of PI 3kinase with the LDM or, more precisely, the GLUT4-containing vesicles. To define further the extent of the insulin-dependent association of PI 3-kinase with these subcellular compartments, we determined its enzymic activity and the localization of its p85 and p110 polypeptides in isolated GLUT4-containing vesicles ('G4V'), in LDM depleted of GLUT4 vesicles ('LDM - G4V'), in complete LDM ('LDM') and in plasma membranes ('PM'). The amounts of each fraction analysed represented an equivalent proportion that was recovered from one $10 \mathrm{~cm}$ dish of adipocytes, i.e. cell equivalents. PI 3-kinase activity was measured in anti(IRS-1) immunoprecipitates from each membrane fraction, to focus on the insulin-dependent effect. In the basal state the PI 3kinase activity ranking was $\mathrm{LDM}>\mathrm{G} 4 \mathrm{~V}>\mathrm{LDM}-\mathrm{G} 4 \mathrm{~V} \approx$ PM (Figure 4A). In the basal state approx. $30 \%$ of the PI 3kinase activity measured in the LDM was associated with the GLUT4-containing vesicles. These results are in close agreement with those of Heller-Harrison et al. [15]. Insulin caused a marked stimulation in PI 3-kinase activity in the LDM and the G4V, and minor changes in the PM and LDM $-\mathrm{G} 4 \mathrm{~V}$. Most but not all of the PI 3-kinase originating from the LDM could be recovered in the G4V and LDM $-\mathrm{G} 4 \mathrm{~V}$ fractions. It is conceivable that some of the activity might have been lost during further manipulation of the samples. A representative immunoblot of the levels of GLUT4 in all four fractions isolated from unstimulated and insulin-stimulated cells shown in Figure 4(B) illustrates the consistency with which GLUT4-containing vesicles were isolated by this vesicle immunoprecipitation protocol. For each fraction, the levels of GLUT4 protein were similar whether isolated from control or insulin-stimulated cells (Figure 4B). This panel also shows that at these short times of insulin action, there was a very small increase in GLUT4 content in the PM fraction.

Insulin stimulates the association of $\mathrm{p} 85 \alpha$ but not $\mathrm{p} 85 \beta$ with IRS-1 [1], but the isoforms of p110 that are responsive to the hormone have not been determined. Figure 4(B) illustrates the immunoblotting results of these fractions in Figure 4(A), for the PI 3-kinase p85 and p110 polypeptides. In the basal state, approx. $40 \%$ of the PI 3-kinase p85 $\alpha$ subunit measured in the LDM was associated with the GLUT4-containing vesicles (Figure 4B). These results are in close agreement with those of HellerHarrison et al. [15]. Insulin treatment for 3.5 min caused an increase in the levels of $\mathrm{p} 85 \alpha$ in the GLUT4-containing vesicles and, consistent with earlier reports, in the complete LDM [13-15]. Furthermore, we detected a parallel gain in the $\mathrm{p} 110 \beta$ isoform of the catalytic subunit on the GLUT4-containing vesicles and in the intact LDM (Figure 4B). In contrast, the $\alpha$ isoform of this subunit was not insulin-responsive (see Figure 5A). Only minor gains in PI 3-kinase $\mathrm{p} 85 \alpha$ and $\mathrm{p} 110 \beta$ subunits were seen in the PM (Figure 4B). Importantly, the insulin-dependent gains in PI 3-kinase activity and its subunits were comparatively small in LDM that were depleted of GLUT4 compartments. The results of Figure 4 suggest that the full, insulin-propagated activation of PI 3-kinase is tightly correlated with the insulin-stimulated levels of tyrosine-phosphorylated IRS-1 $[13,28]$ and might be a combination of translocation of active IRS-1/PI 3-kinase complexes to the GLUT4-containing vesicles and activation of some of the complexes already present on these same vesicles.

The assay of lipid kinase activity in vitro utilizes exogenous PI to produce phosphatidylinositol 3'-phosphate. Two enzymic activities can conceivably generate phosphatidylinositol $3^{\prime}$-phosphate: PI 3-kinase generates PI 3-P, and PI 4-kinase generates PI 4-P. Only the former kinase binds to IRS-1 [29,30]. To establish whether the lipid kinase activity detected on GLUT4-containing vesicles was PI 3-kinase, we tested the sensitivity of this lipid kinase activity to wortmannin, which does not inhibit the membrane-bound form of PI 4-kinase found on the GLUT4containing vesicle [30,31]. The amount of PI kinase associated with the vesicles shown in Table 3 was determined by exposing the purified vesicles directly to the assay mixture (in contrast with the detection of PI 3-kinase activity shown in Figure 4, in which the assay was performed on IRS-1 immunoprecipitates isolated from GLUT4-containing vesicles). This allowed us to detect the content of PI kinase irrespective of the particular insulin receptor substrate. The results from three separate experiments indicated that the insulin-dependent phosphorylation of exogenous lipid by GLUT4-containing vesicles was fully inhibitable by preexposing the vesicles to $100 \mathrm{nM}$ wortmannin for $10 \mathrm{~min}$ before the PI 3-kinase assay (Table 3). The PI lipid kinase activity associated with GLUT4-containing vesicles isolated from unstimulated cells was only minimally affected by wortmannin. PI 4-kinase has been detected in these vesicles and is not affected by insulin stimulation [30]. However, the insulin-stimulated activity was totally inhibited by wortmannin (Table 3). These results indicate that there was insignificant basal activity of PI kinase on the GLUT4-containing vesicles, despite the presence of IRS-1 and the p85 and p110 subunits of PI 3-kinase. Moreover, the PI lipid kinase stimulated by insulin on the GLUT4-containing

\section{Figure 4 Distribution of IRS-1-associated PI 3-kinase activity and the PI 3-kinase $p 85 \alpha$ and $p 110 \beta$ subunits in diverse cellular membrane compartments}

3T3-L1 adipocytes were left either untreated $(-)$ or stimulated with insulin for $3.5 \mathrm{~min}(+)$, after which the LDM plus cytosol fraction and the PM fraction were isolated. The former was separated into two equal samples. From one half of LDM plus cytosol, complete LDM were isolated by centrifugation at $200000 \boldsymbol{g}$ (LDM). From the other half of LDM plus cytosol, GLUT4-containing vesicles (G4V) were immunopurified as described in the Materials and methods section. The supernatant of this immunopurification was centrifuged at $200000 \boldsymbol{g}$ to recover the LDM depleted of GLUT4containing vesicles (LDM - G4V). Equal proportions of each of the four fractions were analysed on SDS/PAGE so that the amounts loaded represented equivalents of the relative complements that each fraction within the intact cells (i.e. $25 \%$ of the samples isolated from each $10 \mathrm{~cm}$-diameter dish were used for each analysis). The fractions were probed for IRS-1-associated PI 3-kinase activity $(\mathbf{A})$ and $\mathrm{p} 85 \alpha$ and $\mathrm{p} 110 \beta$ polypeptides (B). The results of three or four independent experiments were quantified by scanning densitometry. The stimulation of PI 3-kinase by insulin (insulin/basal) was significant in the G4V fraction $(P<0.01)$ and in the LDM fractions $(P<0.05)$. The insulin-stimulated gains of p85 $\alpha$ in the G4V fraction $(P<0.001, n=4)$ and the LDM fraction $(P<0.005, n=4)$ were significant. The insulin-stimulated gains of $p 110 \beta$ in the G4V fraction $(P<0.05, n=6)$ and the LDM fraction $(P<0.01, n=6)$ were significant. Abbreviations: i.b., immunoblotting; PI3P, phosphatidylinositol 3'-phosphate. 
Table 3 Activity in vitro of PI kinase of immunopurified GLUT4-containing vesicles

GLUT4-containing vesicles were immunoisolated from control and insulin-stimulated cells and assayed directly for PI kinase activity towards exogenous PI in the absence or presence of $100 \mathrm{nM}$ wortmannin. The results are expressed in relative units, after assigning a value of 1.0 to the activity in GLUT4-containing vesicles from unstimulated cells assayed in the absence of wortmannin. Results are means \pm S.E.M. for three determinations. ${ }^{*} P<0.05$ compared with untreated control cells (Student's paired $t$ test).

\begin{tabular}{lll}
\hline & \multicolumn{2}{l}{ PI kinase activity (relative to control) } \\
\cline { 2 - 3 } & Control & Insulin-stimulated \\
\hline - Wortmannin & 1.0 & $2.9 \pm 0.5^{*}$ \\
+ Wortmannin & $0.8 \pm 0.2$ & $1.1 \pm 0.3$ \\
\hline
\end{tabular}

A

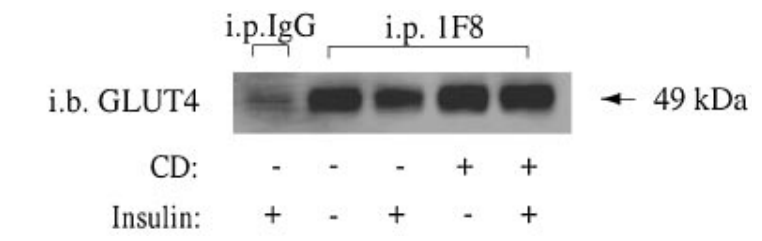

B

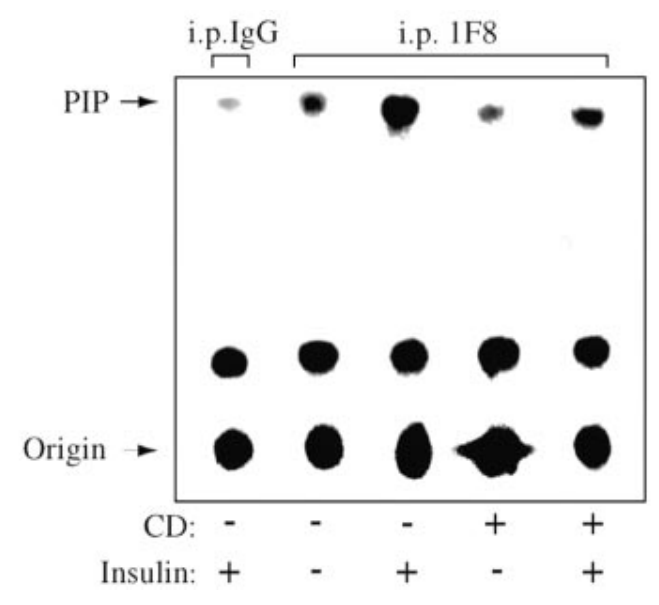

Figure 5 Effects of insulin and CD on GLUT4 vesicle-associated PI 3kinase activity

GLUT4 containing-vesicles were immunopurifed (i.p.) from LDM of untreated, insulin-stimulated (3.5 min), CD-pretreated, or CD-pretreated insulin-stimulated adipocytes and used for immunoblotting or for direct assay of PI 3-kinase activity. Non-immune murine IgG was used as control to analyse non-specifically sedimented membranes from the LDM prepared from insulin-treated cells. (A) Recovery of vesicles was estimated by immunoblotting (i.b.) the samples for GLUT4. The yield of immunopurified GLUT4-containing vesicles from the LDM was substantial, and not affected by any of the treatments: $90 \pm 5 \%$ for control cells, $88 \pm 6 \%$ for insulin-stimulated cells, $88 \pm 5 \%$ for CD-pretreated cells, and $90 \pm 5 \%$ for CDpretreated insulin-stimulated cells. (B) A representative autoradiograph is shown of ${ }^{32} \mathrm{P}$-labelled phosphatidylinositol 3'-phosphate (PIP) separated by TLC in three separate experiments.

vesicles was only PI 3-kinase. A comparison of the results in Figure 4 and Table 3 indicates that insulin caused a 2.2-fold stimulation of PI kinase activity associated with GLUT4-containing vesicles, and the wortmannin-sensitive component of this activity was stimulated 3.2 -fold above the respective values in vesicles from unstimulated cells.
Table 4 Effects of insulin and CD on GLUT4 vesicle-associated PI kinase activity

GLUT4-containing vesicles were immunopurifed from LDM of untreated, insulin-stimulated (3.5 min), CD-pretreated (CD) or CD-pretreated insulin-stimulated (CD + insulin) adipocytes and used for the direct measurement of PI kinase activity on the vesicles. Non-immune murine IgG (NI-lgG) was used as control to analyse the PI kinase activity associated with the non-specific sedimentation of membranes from the LDM prepared from insulin-treated cells. PI kinase activity was measured directly on the immunopurified vesicles as described in the Materials and methods section and the legend to Figure 5 . The radioactivity associated with phosphatidylinositol 3'-phosphate (PIP) spots from TLC plates like those shown in Figure 5(B) was determined by scraping off the spots from the plates and scintillation counting. The results were calculated as the means of c.p.m. detected in the PIP spots of three separate experiments.The untreated value was set at 1.0 and all other values were calculated relative to this. The PI kinase activity associated with GLUT4-containing vesicles from CD-pretreated insulin-stimulated cells were significantly different $(P<0.01$, ANOVA) from the activity associated with GLUT4-containing vesicles from insulin-stimulated cells not pretreated with $C D$.

PI kinase activity (relative to untreated)

\begin{tabular}{lll}
\hline Anti-GLUT4-pelleted vesicles & NI-lgG-pelleted vesicles \\
\hline Untreated $\quad$ Insulin $\quad$ CD & CD + insulin
\end{tabular}

PI kinase activity $1.00 \pm 0.03 \quad 2.15 \pm 0.22 \quad 0.86 \pm 0.081 \quad 1.25 \pm 0.11 \quad 0.52 \pm 0.080$

We next examined whether pretreatment with $\mathrm{CD}$, which disassembles actin filaments and reduces the stimulation of glucose transport, affected the gain in PI 3-kinase activity and its subunits on the GLUT4-containing vesicles. For these experiments it was important to ascertain that equal amounts of GLUT4-containing vesicles were compared between the different cell treatments. Immunopurification of GLUT4-containing vesicles from LDM was substantial and was not influenced by the prior treatment that the cells received. In three experiments, $90 \pm 5 \%$ of the GLUT4-containing vesicles were immunoprecipitated from untreated cells; $88 \pm 6 \%$ from insulinstimulated cells; $88 \pm 5 \%$ from CD pretreated cells; and $90 \pm 5 \%$ from CD pretreated insulin-stimulated cells (data given as means \pm S.E.M.) (Figure 5A). In contrast, non-immune mouse IgG removed only $13 \pm 4 \%$ of the GLUT4-containing vesicles from the LDM/cytosol starting material (Figure 5A). Thus the GLUT4 vesicle isolation protocol was consistent and unaffected by the prior treatments that the cells had received. The supernatants of these immunoprecipitations (from which LDM $-\mathrm{G} 4 \mathrm{~V}$ were isolated) contained a very low level of GLUT4 protein and this amount did not differ between samples $(10 \pm 1 \%$ in untreated samples; $12 \pm 1 \%$ in insulin-stimulated samples; $12 \pm 2 \%$ in CD-pretreated samples; $10 \pm 1 \%$ in CD-pretreated and insulin-stimulated samples).

Figure 5(B) illustrates results from three independent experiments in which GLUT4-containing vesicles were assayed for PI kinase activity after $3.5 \mathrm{~min}$ of insulin stimulation of control and CD-pretreated cells. The amount of PI kinase associated with the vesicles was determined by exposing the purified vesicles directly to the assay mixture. Insulin caused a marked activation or association of PI kinase activity with the GLUT4-containing vesicles (Figure 5B), which from Figure 4 we know was PI 3-kinase. Non-immune IgG did not immunoprecipitate a substantial amount of PI kinase activity from the LDM membranes (Figure 5B). Importantly, the insulin-dependent PI 3-kinase activity associated with the GLUT4-containing vesicles from CD-pretreated cells was lower (by $42 \%, P<0.002$ ) in vesicles than in untreated cells. The net gain above basal 


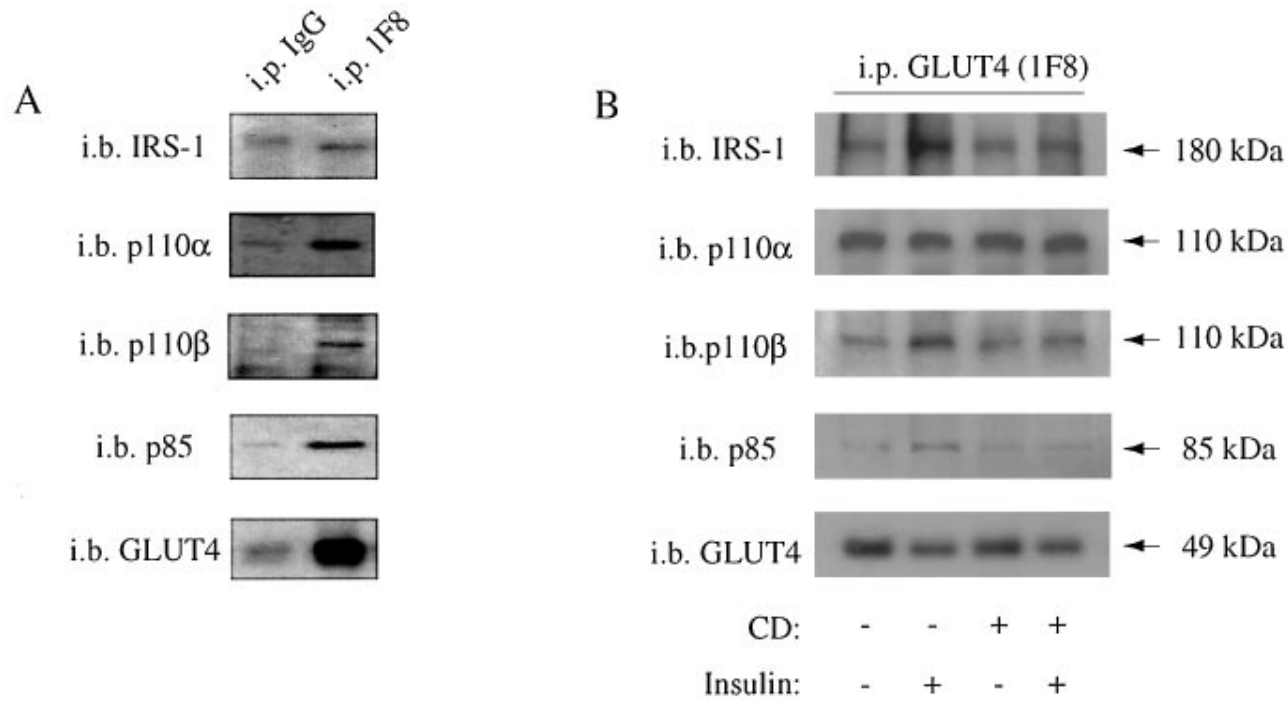

\section{$\mathbf{C}$}
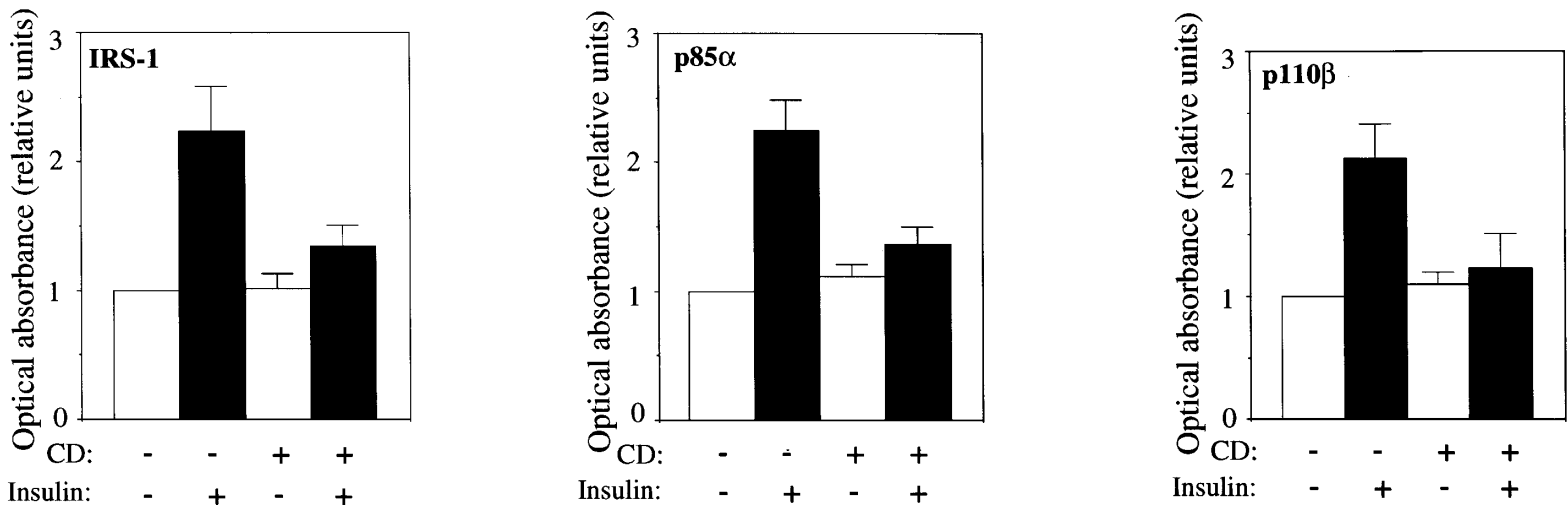

Figure 6 Effects of insulin and CD on GLUT4-containing vesicle associated IRS-1 and PI 3-kinase polypeptides

GLUT4-containing vesicles were immunopurified (i.p.) from LDM of 3T3-L1 adipocytes with monoclonal anti-GLUT4 antibody (1F8) as outlined in the Materials and methods section. The membrane fractions were then analysed by SDS/PAGE and immunoblotted (i.b.) with antibodies to the proteins IRS-1 and the PI 3-kinase subunits p85 $\alpha$, p110 $\beta$, p110 $\alpha$ and the GLUT4 protein. (A) To determine the specificity of our vesicle immunoisolation protocol, non-immune mouse immunoglobulin (IgG) or anti-glucose transporter monoclonal antibody (1F8) was used to immunoprecipitate vesicles from LDM of untreated cells and immunoblotted for GLUT4, IRS-1, p85 $\alpha, \mathrm{p} 110 \beta$ and $\mathrm{p} 110 \alpha$. Representative immunoblots are shown from two or three independent experiments for each protein. (B) Representative immunoblots are illustrated of the same set of proteins (analysed in A) associated with GLUT4-containing vesicles isolated from untreated, insulin-stimulated (3.5 min), CDpretreated, and CD-pretreated insulin-stimulated cells, as indicated. (C) The mean of three or four independent experiments showing the relative abundance of the IRS-1, p85 $\alpha$ and p110 $\beta$ in the same fractions illustrated in (A). The results presented were normalized to the value in the corresponding fraction from untreated cells. The net gain $(\Delta)$ caused by insulin in IRS-1 from CD-pretreated cells was significantly different $(P<0.03)$ from that in untreated cells. The net gain $(\Delta)$ caused by insulin in p85 $\alpha$ polypeptide and p110 $\beta$ polypeptide from CD-pretreated cells was significantly different $(P<0.002$ and $P<0.05$ respectively) from that in untreated cells.

activity $(\Delta)$ caused by insulin was also lower in CD-pretreated cells (by $66 \%, P<0.01$ ) (Table 4 ). These results indicate that full activation or association of PI 3-kinase activity with the GLUT4-containing vesicles correlates with the existence of fully polymerized actin filaments.

To analyse further the effects of $\mathrm{CD}$ on the distribution of PI 3-kinase subunits and associated proteins, we studied the presence of IRS-1, p110 $\alpha$, p110 $\beta$ and p85 $\alpha$, and GLUT4 on the GLUT4containing vesicles isolated from control, insulin-stimulated, CDpretreated and CD-pretreated insulin-stimulated cells. Control experiments were performed with non-immune $\mathrm{IgG}$ to sediment any membranes that bound non-specifically in our vesicle isolation protocol (Figure 6A). None of these proteins were found to any significant degree in the control immunoprecipitates, whereas they were detected on GLUT4 immunopurified vesicles (Figure 6A). Insulin rapidly increased the levels of IRS-1, p85 $\alpha$ and $\mathrm{p} 110 \beta$, but not $\mathrm{p} 110 \alpha$, on the GLUT4-containing vesicles
(Figure 6B). CD treatment of adipocytes had no effect on the basal levels of these molecules on the GLUT4-containing vesicles, but CD significantly diminished the insulin-dependent associations of IRS-1, p85 $\alpha$ and $\mathrm{p} 110 \beta$ with the GLUT4-containing vesicles (Figure 6B). The lack of changes in the levels of p110 $\alpha$ in response to insulin suggests that not all proteins were equally affected. The levels of each protein were quantified by scanning densitometry of autoradiographs of immunoblots for each protein (Figure 6C). The results from the scans of at least three or four independent experiments were expressed in relative units with the basal level of each protein assigned a value of 1.0. The inhibition by CD of the insulin-stimulated recruitment of IRS-1, $\mathrm{p} 85 \alpha$ and $\mathrm{p} 110 \beta$ was significant in each case (see the legend to Figure $6 \mathrm{C}$ for a statistical analysis). These results suggest that insulin causes the translocation of PI 3-kinase to GLUT4containing vesicles and that disassembly of actin filaments by CD prevents this translocation to a significant degree. 


\section{DISCUSSION}

\section{Association of PI 3-kinase with GLUT4-containing vesicles}

The stimulation of glucose transport, largely accounted for by the translocation of glucose transporters to the cell surface, is abolished by the inhibitors of PI 3-kinase wortmannin and LY294002 in fat and muscle cells [3-5]. PI 3-kinase activation seems to relay the insulin signal rather than to facilitate vesicle fusion, because activation of this enzyme is not required by other stimuli that also stimulate the translocation of glucose transporters to the cell surface, such as exercise and hypoxia [32], dinitrophenol [33], phorbol esters [34] and okadaic acid [35]. Thus it is important to define how the insulin-stimulated PI 3kinase triggers vesicle translocation.

Several studies suggest that an increase in PI 3-kinase enzymic activity is not sufficient to bring about the stimulation of glucose transport, because other agents that markedly elevate the endogenous kinase activity, e.g. PDGF, interleukin 4 and epidermal growth factor, do not increase the rate of glucose uptake $[10,11,36]$. This compelling evidence suggests that ancillary mechanisms triggered by insulin are required. One such factor might be the localization of the activated enzyme. In 3T3-L1 adipocytes, most of the IRS- 1 is localized to the LDM in the basal state [37]. Within minutes of insulin administration, IRS-1 in the LDM is rapidly phosphorylated on tyrosine residues by the insulin receptor [37,38]. Interestingly, the time course of this event is tightly coupled to the translocation of GLUT4 proteins from the LDM [38]. Moreover IRS-1-associated PI 3-kinase activity and immunoreactive p85 increase in LDM $[13,14]$ and in GLUT4-containing vesicles [15]. In contrast, PDGF does not induce the redistribution of PI 3-kinase from the cytosol to the LDM $[11,14]$. However, three studies have proposed that supraphysiological elevation of PI 3-kinase activity by the overexpression of the enzyme can stimulate glucose uptake [7-9]. It is conceivable that under such conditions the kinase could populate the vesicles containing glucose transporters in the absence of an insulin-derived signal, overriding the natural pathway of PI 3-kinase association to IRS-1 and translocation to the vesicles. In fact, one study demonstrates that a significant proportion of the constitutively activated p110 is found in the LDM of rat adipocytes [9]. This would lend support to the hypothesis that, under normal conditions, only insulin causes the translocation of PI 3-kinase to GLUT4 vesicles, thereby stimulating the translocation of GLUT4-containing vesicles to the cell surface and, hence, glucose transport.

Here we report that insulin increases the PI 3-kinase activity and the immunoreactivity of $\mathrm{p} 85 \alpha$ on the GLUT4-containing vesicle isolated from the LDM of 3T3-L1 adipocytes, in a manner very much like that reported by Heller-Harrison et al. [15]. We extend these findings by demonstrating that insulin treatment also causes a marked increase in immunodetectable IRS- 1 and the $\mathrm{p} 110 \beta$ (but not the $\mathrm{p} 110 \alpha$ ) catalytic subunit of PI 3-kinase with the GLUT4-containing vesicles. Because wortmannin does not inhibit the redistribution of PI 3-kinase to the LDM [14], the well-recognized ability of wortmannin to prevent the stimulation of glucose transport by insulin might be due to the inhibition of PI 3-kinase in the complex of IRS-1, p85 $\alpha$ and p1 $10 \beta$ after it has associated with the GLUT4-containing vesicles. To our knowledge this is the first demonstration of migration of the catalytic subunit of PI 3-kinase to a GLUT4-containing vesicle in response to insulin. A previous study suggested that the LDM vesicles that carry the bulk of the GLUT4 protein are distinct from those that carry IRS-1 and PI 3-kinase [28]. However, insulin treatments were performed for $10 \mathrm{~min}$, at a time when most of the translocatable GLUT4-containing vesicles have left the LDM. Those that remain might be refractory to translocation and are perhaps unable to interact with the IRS1-PI 3-kinase complex. PI 3-kinase association with GLUT4containing vesicles must occur at earlier times after addition of insulin to the cells, before the full activation of glucose transport [15]. Interestingly, the density of the GLUT4-containing vesicles in the LDM begins to shift after a $3.5 \mathrm{~min}$ stimulation with insulin compared with GLUT4-containing vesicles from unstimulated cells [15]. This shift in density might be an indicator of the separation of GLUT4 and PI 3-kinase in the LDM.

\section{Effect of disruption of actin filaments on PI 3-kinase activity and distribution}

The main finding of this study is that the disassembly of a significant number of actin filaments with $C D$ coincided with a profound decrease in the insulin-dependent gain in PI 3-kinase activity on GLUT4-containing vesicles. Concomitantly, CD pretreatment significantly diminished the increase in the insulindependent content of IRS- 1 and the PI 3-kinase p85 $\alpha$ and p110 $\beta$ subunits on the GLUT4-containing vesicles. These results suggest that an intact actin network is at least permissive for the arrival of activated PI 3-kinase at these vesicles. In keeping with this interpretation, CD pretreatment did not prevent the translocation of GLUT1 and GLUT4 glucose transporters and the stimulation of glucose uptake by dinitrophenol in L6 muscle cells [33], a phenomenon that, as stated above, does not require PI 3-kinase signalling. These results support the notion that $\mathrm{CD}$ does not interfere with all membrane vesicle traffic events to the PM but rather that its action is correlated with the need for PI 3-kinase activation, and in this way it inhibits the relay of information from the insulin receptor to the GLUT4 vesicles.

In contrast with its ability to prevent the insulin-dependent gain in PI 3-kinase activity on GLUT4-containing vesicles, CD pretreatment did not affect the insulin-dependent phosphorylation of IRS-1, its binding to the p85 subunit of PI 3-kinase or the activation of PI 3-kinase. These results suggest that the actin network does not alter the formation of the molecular complexes that lead to activation of the enzyme. In fact there is a precedent for a role of actin filaments in the propagation of intracellular signals. In particular, disruption of the actin cytoskeleton with $\mathrm{CD}$ prevented the tyrosine phosphorylation of several proteins in focal adhesions induced by growth factors and other mitogens [39]. The interaction of actin filaments with GLUT4-containing vesicles might require linking proteins such as spectrin. It is particularly compelling that spectrin is present on GLUT4containing vesicles of L6 myotubes [17] and we have preliminary results that spectrin is on GLUT4-containing vesicles of 3T3-L1 adipocytes (Q. Wang, T. Tsakiridis and A. Klip, unpublished work). It has recently been proposed that spectrin might facilitate the formation of distinct membrane domains and contribute to membrane protein sorting [40]. Whether the presence of spectrin is crucial for the arrival of PI 3-kinase to the GLUT4-containing vesicles should be an interesting focus for future investigation.

\section{Inhibition of stimulation of glucose transport and vesicle traffic on disassembly of actin fibres}

Given the prevention of arrival of PI 3-kinase activity at the GLUT4-containing vesicles on the disassembly of actin fibres by $\mathrm{CD}$, we explored the consequence of this treatment on glucose transport. Whereas the basal rate of glucose uptake was not affected, there was a significant decrease in the ability of insulin to stimulate glucose uptake. The correlation between disassembly of the actin cytoskeleton and the inhibition of insulin-stimulated 
glucose uptake was further established by the use of LB, a chemical structurally unrelated to $\mathrm{CD}$ that also promotes actin filament disassembly. Both drugs had similar inhibitory effects on the action of insulin on glucose uptake. The underlying mechanism seems to be the prevention of translocation of the GLUT1 and GLUT4 glucose transporters to the cell surface, because the magnitude of the decrement of glucose transport matched that of the glucose transporter translocation.

Three possible models could explain why the inhibition of insulin-stimulated glucose uptake was only approx. 50\%. First, it is possible that, under the conditions used, short actin filaments remained and contributed to the remaining activation of glucose transport. Indeed, CD did not eliminate the existence of polymerized actin (as indicated by the phalloidin staining that remained); rather, it decreased the number and length of actin filaments. Secondly, it is conceivable that cytoskeletal elements other than actin filaments contributed to the relay of insulin signals needed for stimulation of glucose uptake. Thirdly, it is plausible that only one intracellular compartment was affected by the CD pretreatment. Martin et al. [41] have reported that approx. $40 \%$ of the GLUT4 populates an endocytic GLUT1 compartment, whereas the rest is associated with a selective compartment. In our hands, $42 \pm 2 \%(n=4)$ of the GLUT1 transporter content of the LDM co-purified with the GLUT4containing vesicles. It is well established that both GLUT1 and GLUT4 translocate to the PM in response to insulin in 3T3-L1 adipocytes, and it is possible that insulin mobilizes both intracellular compartments, albeit to different extents. The endocytic compartment was proposed to be enriched in the v-SNARE protein cellubrevin and the selective compartment enriched in the v-SNARE protein VAMP-2 [41,42], and both of these proteins are translocated to the PM in response to insulin $[24,41]$. In Figure 2 we observed that the CD pretreatment decreased the translocation to the PM of both GLUT1 and GLUT4, and in experiments not shown we observed that the drug also decreased the translocation of cellubrevin and VAMP-2. These results suggest that CD pretreatment might decrease the arrival of PI 3kinase subunits to both the endocytic and the selective compartments, but further studies are required to characterize more accurately the two compartments and indeed their requirement for PI 3-kinase.

\section{Effect of insulin on actin filaments}

The actin cytoskeleton is a dynamic structure that can undergo several levels of organization. In resting cells, actin filaments constitute an actin cortex running parallel to the spectrin membrane skeleton and capped actin filaments distributed through the cell body. Stimulation of cells with integrin ligands or certain growth factors reorganizes filaments so they abut the membrane to form membrane ruffles/lamellipodia, as well as to bundle with myosin to form stress fibres. The formation of ruffles/lamellipodia is under the control of the small GTPbinding protein Racl. Activated Racl in turn leads to the activation of the small GTP-binding protein Rho, which controls the formation of stress fibres and thus of focal adhesion plaques [43]. Insulin has been reported to promote actin polymerization [44], formation of ruffles/lamellipodia through Rac1 activation $[45,46]$ and emergence of stress fibres $[18,19]$. In the present study we observed that insulin alters the organization of the actin filaments to produce membrane ruffles in 3T3-L1 adipocytes and that the disassembly of actin filaments by CD precludes most of their insulin-dependent rearrangement.

A question raised by this study is whether the actin-filamentassisted activation of PI 3-kinase on the GLUT4-containing vesicles depends on: (1) the existence of actin filaments within the cell body and along the membrane skeleton, (2) the insulininduced organization of cortical actin filaments in lamellipodia/ruffles, or (3) the formation of stress fibres. CD treatment interferes with all these levels of cytoskeletal organization. A recent study showed that a dominant-inhibitory Rac1 mutant prevented lamellipodia formation but allowed the stimulation of glucose uptake in 3T3-L1 adipocytes [47]. It is possible that introduction of the dominant inhibitory Racl would also prevent the formation of stress fibres via Racl-activated Rho, although this was not addressed directly. Hence it is possible that the actin filaments that participate in the mobilization of PI 3-kinase to the GLUT4-containing vesicles do not arise from membrane ruffles or stress fibres. Instead, actin filaments, not necessarily the insulin-induced changes in their organization, might be the required structure for this relocalization of PI 3-kinase.

In conclusion, insulin induces the reorganization of actin filaments in 3T3-L1 adipocytes. Significant disassembly of actin filaments does not alter events proximal to the insulin receptor. Instead it decreases the increase in PI 3-kinase activity and the gain in $\mathrm{p} 85 \alpha$ and $\mathrm{p} 110 \beta$ subunits on GLUT4-containing vesicles. In parallel, the translocation of glucose transporters to the cell surface and the stimulation of glucose transport are decreased. These correlative results suggest that an intact actin network is required for the complete glucose transport response, and highlight the importance of the subcellular localization of activated PI 3-kinase in the control of insulin-stimulated glucose uptake.

We thank Dr. C. R. Kahn and Dr. K. J. Sweadner for the antibodies to IRS-1 and for the $\alpha$-subunit of the $\mathrm{Na}^{+} / \mathrm{K}^{+}$-ATPase respectively; A. Volchuk and Dr. S. Grinstein for useful comments throughout this study; and Dr. F. Chen for contribution to one of the experiments. This work was supported by grant MT-7307 from the Medical Research Council to A.K. Q.W. was supported by a post-doctoral fellowship from the Hospital for Sick Children Training Committee. T.T. was supported by an Ontario Graduate Scholarship and a Medical Research Council Fellowship.

\section{REFERENCES}

1 Baltensperger, K., Kozma, L. M., Jaspers, S. R. and Czech, M. P. (1994) J. Biol. Chem. 269, 28937-28946

2 Okada, T., Kawano, Y., Sakakibara, T., Hazeki, O. and Ui, M. (1994) J. Biol. Chem. 269, 3568-3573

3 Cheatham, B., Vlahos, C. J., Cheatham, L., Wang, L., Blenis, J. and Kahn, C. R. (1994) Mol. Cell. Biol. 14, 4902-4911

4 Tsakiridis, T., McDowell, H. E., Walker, T., Downes, C. P., Hundal, H. S., Vranic, M. and Klip, A. (1995) Endocrinology (Baltimore) 136, 4315-4322

5 Kotani, K., Carozzi, A. J., Sakaue, H., Hara, K., Robinson, L. J., Clark, S. F., Yonezawa, K., James, D. E. and Kasuga, M. (1995) Biochem. Biophys. Res. Commun. 209, 343-348

6 Quon, M. J., Chen, H., Ing, B. L., Liu, M.-L., Zarnowski, M. J., Yonezawa, K., Kasuga, M., Cushman, S. W. and Taylor, S. I. (1995) Mol. Cell. Biol. 15, 5403-5411

7 Katagiri, H., Asano, T., Ishihara, H., Inukai, M., Yazaki, Y. and Oka, Y. (1996) J. Biol. Chem. 271, 16987-16990

8 Martin, S. S., Haruta, T., Morris, A., Klippel, A., Williams, L. T. and Olefsky, J. M. (1996) J. Biol. Chem. 271, 17605-17608

9 Tanti, J. F., Gremeaux, T., Grillo, S., Calleja, V., Klippel, A., Williams, L. T., Van Obberghen, E. and Le Marchand-Brustel, Y. (1996) J. Biol. Chem. 271, 25227-25232

10 Isakoff, S. J., Taha, C., Rose, E., Marcusohn, J., Klip, A. and Skolnik, E. Y. (1995) Proc. Natl. Acad. Sci. U.S.A. 92, 10247-10251

11 Ricort, J.-M., Tanti, J.-F., Van Obberghen, E. and Le Marchand-Brustel, Y. (1996) Eur. J. Biochem. 239, 17-22

12 Herbst, J. J., Andrews, G. C., Contillo, L. G., Singleton, D. H., Genereux, P. E., Gibbs, E. M. and Lienhard, G. E. (1995) J. Biol. Chem. 270, 26000-26005

13 Yang, J., Clarke, J. F., Ester, C. J., Young, P. W., Kasuga, M. and Holman, G. D. (1996) Biochem. J. 313, 125-131

14 Nave, B. T., Haigh, R. J., Hayward, A. C., Siddle, K. and Shepherd, P. R. (1996) Biochem. J. 318, 55-60 
15 Heller-Harrison, R. A., Morin, M., Guilherme, A. and Czech, M. P. (1996) J. Biol. Chem. 271, 10200-10204

16 Nobes, C. D., Hawkins, P., Stephens, L. and Hall, A. (1995) J. Cell Sci. 108 225-233

17 Tsakiridis, T., Vranic, M. and Klip, A. (1994) J. Biol. Chem. 269, 29934-29942

18 Ridley, A. J. and Hall, A. (1992) Cell 70, 389-399

19 Berfield, A. K., Raugi, G. J. and Abeass, C. K. (1996) J. Histochem. Cytochem. 44 91-101

20 Felsenfeld, D. P. and Sweadner, K. J. (1988) J. Biol. Chem. 263, 10932-10942

21 Frost, S. C. and Lane, M. D. (1985) J. Biol. Chem. 260, 2646-2652

22 Volchuk, A., Wang, Q., Ewart, H. S., Liu, Z., He, L., Bennett, M. K. and Klip, A. (1996) Mol. Biol. Cell 7, 1075-1082

23 Laemmli, U. K. (1970) Nature (London) 227, 680-685

24 Volchuk, A., Sargeant, R., Sumitani, S., Liu, Z., He, L. and Klip, A. (1995) J. Biol. Chem. 270, 8233-8240

25 Laurie, S. M., Cain, C. C., Lienhard, G. E. and Castle, D. (1993) J. Biol. Chem. 268, 19110-19117

26 Spector, I., Shochet, R., Blasberger, D. and Kashman, Y. (1989) Cell Motil. Cytoskel. 13, 127-144

27 Calderhead, D. M., Kitagawa, K., Tanner, L. I., Holman, G. D. and Lienhard, G. E. (1990) J. Biol. Chem. 265, 13801-13808

28 Kelly, K. and Ruderman, N. (1993) J. Biol. Chem. 268, 4391-4398

29 White, M. F. and Kahn, C. R. (1994) J. Biol. Chem. 269, 1-4

30 Del Vecchio, R. L. and Pilch, P. F. (1991) J. Biol. Chem. 266, 13278-13283

31 Nakanishi, S., Catt, K. J. and Balla, T. (1995) Proc. Natl. Acad. Sci. U.S.A. 92 , $5317-5321$

Received 15 December 1997/5 February 1998; accepted 13 February 1998
32 Coderre, L., Kandror, K. V., Vallega, G. and Pilch, P. F. (1995) J. Biol. Chem. 270 , 27584-27588

33 Tsakiridis, T., Vranic, M. and Klip, A. (1995) Biochem. J. 309, 1-5

34 Ahmad, F., Li, P.-M., Meyerovitch, J. and Golstein, B. J. (1995) J. Biol. Chem. 270 20503-20508

35 Rampal, A. L., Jhun, B. H., Kim, S., Liu, H., Manka, M., Lachaal, M., Spangler, R. A. and Jung, C. Y. (1995) J. Biol. Chem. 270, 3938-3943

36 Wiese, R. J., Mastick, C. C., Lazar, D. F. and Saltiel, A. R. (1995) J. Biol. Chem. 270 3442-3446

37 Heller-Harrison, R. A., Morin, M. and Czech, M. P. (1995) J. Biol. Chem. 270, 24442-24450

38 Kublaoui, B., Lee, J. and Pilch, P. F. (1995) J. Biol. Chem. 270, 59-65

39 Lacerda, H. M., Lax, A. J. and Rozengurt, E. (1996) J. Biol. Chem. 271, 439-445

40 Beck, K. A. and Nelson, W. J. (1996) Am. J. Physiol. 270, C1263-C1270

41 Martin, S., Tellman, J., Livingstone, C., Slot, J. W., Gould, G. W. and James, D. E. (1996) J. Cell Biol. 134, 625-635

42 Cheatham, B., Volchuk, A., Kahn, C. R., Wang, L., Rhodes, C. J. and Klip, A. (1996) Proc. Natl. Acad. Sci. U.S.A. 93, 15169-15173

43 Hotchin, N. A. and Hall, A. (1995) J. Cell Biol. 131, 1857-1865

44 Kirkeeide, E. K., Pryme, I. F. and Vedeler, A. (1993) Int. J. Biochem. 25, 853-864

45 Nobes, C. D. and Hall, A. (1995) Cell 81, 53-62

46 Kotani, K., Hara, K., Kotani, K., Yonezawa, K. and Kasuga, M. (1995) Biochem. Biophys. Res. Commun. 208, 985-990

47 Marcusohn, J., Isakoff, S. J., Rose, E., Symons, M. and Skolnik, E. Y. (1995) Curr. Biol. 5, 1296-1302 